Journal of Organometallic Chemistry, 243 (1983) 179-189

Elsevier Sequoia S.A., Lausanne - Printed in The Netherlands

\title{
UNTERSUCHUNGEN ZUR STRUKTUR UND STEREOCHEMIE DER ADDITIONSPRODUKTE VON NUKLEOPHILEN AN VON $N$-METHYLBENZIMIDCHLORID ABGELEITETE CARBENCHELATKOMPLEXE
}

HENRI BRUNNER, JOACHIM WACHTER,

Institut für Anorganische Chemie der Universität Regensburg, Universitätsstrasse 31, D-8400 Regensburg (B.R.D.)

IVAN BERNAL, GEORGE M. REISNER

Department of Chemistry, University of Houston, Houston, Texas 77004 (U.S.A.)

und REINHARD BENN

Max-Planck-Institut für Kohlenforschung, Kaiser-Wilhelm-Platz I, D-4330 Mülheim a.d. Ruhr (B.R.D.) (Eingegangen den 24. September 1982)

\section{Summary}

In the reaction of $\left[\mathrm{C}_{5} \mathrm{H}_{5}(\mathrm{CO})_{2} \mathrm{MoC}\left(\mathrm{C}_{6} \mathrm{H}_{5}\right) \mathrm{N}\left(\mathrm{CH}_{3}\right) \mathrm{C}\left(\mathrm{C}_{6} \mathrm{H}_{5}\right) \mathrm{N}\left(\mathrm{CH}_{3}\right)\right] \mathrm{PF}_{6}$ (I) with $\mathrm{C}_{6} \mathrm{H}_{5} \mathrm{MgBr}$ the phenyl group adds to the carbene carbon atom giving the neutral complex $\mathrm{C}_{5} \mathrm{H}_{5}(\mathrm{CO})_{2} \mathrm{MoC}_{6}\left(\mathrm{C}_{6} \mathrm{H}_{5}\right)_{2} \mathrm{~N}\left(\mathrm{CH}_{3}\right) \mathrm{C}\left(\mathrm{C}_{6} \mathrm{H}_{5}\right) \mathrm{N}\left(\mathrm{CH}_{3}\right)(\mathrm{V})$. $\mathrm{V}$ crystallizes in the space group $P 2_{1} / c$ with the cell constants $a 11.803(5), b 12.114(6), c 17.198(6) \AA$, $\beta 96.61(3)^{0}, V 2431.2 \AA^{3}, Z=4, \rho_{\text {calc }} 1.45 \mathrm{~g} \mathrm{~cm}^{-3}$. For 2979 reflections the $R$ value is 5.1, $R_{k}=4.8$. The stereoisomers IIIA and IIIB, formed in the $\mathrm{H}^{-}$addition to I, are ${ }^{1} \mathrm{H}$ NMR spectroscopically investigated by means of Nuclear-Overhauser-Effect Difference Spectroscopy in order to elucidate the orientation of the incoming $\mathbf{H}$ substituent with respect to the $\mathrm{C}_{5} \mathrm{H}_{5}$ group.

\section{Zusammenfassung}

Die Reaktion von $\left[\mathrm{C}_{5} \mathrm{H}_{5}(\mathrm{CO})_{2} \mathrm{MoC}\left(\mathrm{C}_{6} \mathrm{H}_{5}\right) \mathrm{N}\left(\mathrm{CH}_{3}\right) \mathrm{C}\left(\mathrm{C}_{6} \mathrm{H}_{5}\right) \mathrm{N}\left(\mathrm{CH}_{3}\right)\right] \mathrm{PF}_{6}$ (I) mit $\mathrm{C}_{6} \mathrm{H}_{5} \mathrm{MgBr}$ ergibt unter Addition des Phenylrestes an den Carbenkohlenstoff den neutralen Komplex $\mathrm{C}_{5} \mathrm{H}_{5}(\mathrm{CO})_{2} \mathrm{MoC}\left(\mathrm{C}_{6} \mathrm{H}_{5}\right)_{2} \mathrm{~N}\left(\mathrm{CH}_{3}\right) \mathrm{C}\left(\mathrm{C}_{6} \mathrm{H}_{5}\right) \mathrm{N}\left(\mathrm{CH}_{3}\right)$ (V). V kristallisiert in der Raumgruppe $P 2_{1} / c$ mit den Zellkonstanten $a 11.803(5), b 12.114(6)$, c 17.198(6) $\AA, \beta 96.61(3)^{0}, V 2431.2 \AA^{3}, Z=4, \rho_{\text {ber }} 1.45 \mathrm{~g} \mathrm{~cm}^{-3}$. Für 2979 Reflexe ist der $R$-Wert 5.1, $R_{k}=4.8$. Die bei der $\mathrm{H}^{-}$-Addition an $\mathrm{I}$ entstehenden Stereoisomere IIIA und IIIh werden 'H-NMR-spektroskopisch anhand von Kern-Overhauser-Effekt-Differenzspektroskopie auf die Orientierung des neu eintretenden $\mathrm{H}$-Substituenten bezüglich des $\mathrm{C}_{5} \mathrm{H}_{5}$-Restes untersucht. 


\section{Einleitung}

Die Reduktion der Carbenchelatkomplexe $\left[\mathrm{C}_{5} \mathrm{H}_{5}(\mathrm{CO})_{x} \mathrm{MC( \textrm {C } _ { 6 } \mathrm { H } _ { 5 } )}\right.$ -

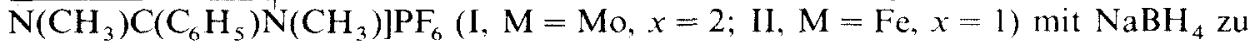
den neutralen Verbindungen III und IV (Schema 1) beansprucht aufgrund ihres nahezu stereospezifischen Ablaufs besonderes Interesse [1]. Je nach der Orientierung des neu eintretenden $\mathrm{H}^{-}$-Substituenten bezüglich des $\mathrm{C}_{5} \mathrm{H}_{5}$-Restes entstehen zwei verschiedene Isomere A und B (Schema 2). Bisher wurde vorausgesetzt, dass bei der Reaktion der Chelatfünfring erhalten bleibt. Um Ringverengungsreaktionen [2] sicher auszuschliessen, schien eine Absicherung durch Röntgenstrukturanalyse wünschenswert. Als hierzu geeignet erwies sich das Phenyladdukt $V$, über dessen Synthese und Röntgenstrukturbestimmung wir hier berichten. Nach der Lösung des strukturellen Problems ist der Weg frei für die Klärung des stereochemischen Ablaufs der Hydridaddition auf ${ }^{1}$ H-NMR-spektroskopischem Weg. Hierzu haben wir Messungen des Kern-Overhauser-Effektes (NOE) am Mo-Komplex III durchgeführt. NOE-Differenzspektren sind bereits mit Erfolg bei der Konformationsanalyse optisch aktiver $\mathrm{C}_{5} \mathrm{H}_{5}(\mathrm{CO})_{2}$ Mo-Amidinat-Komplexe angewendet worden [3]. Über Details der NOE-Differenzspektroskopie wurde bereits früher berichtet [4].

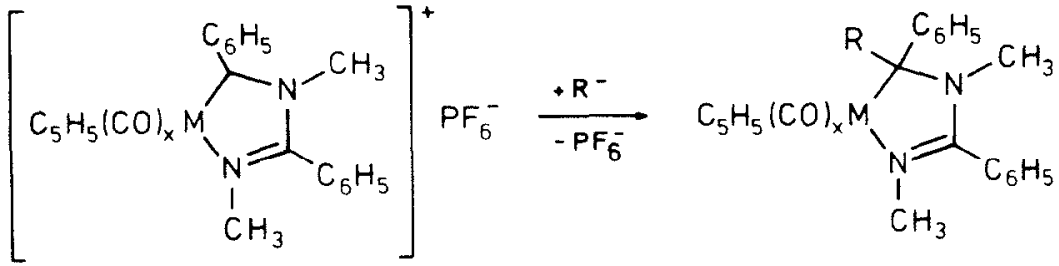

$$
\begin{aligned}
& M=M_{0}(x=2): I \\
& M=F e(x=1): \Pi
\end{aligned}
$$

\begin{tabular}{c|cc}
$R$ & $H$ & $C_{6} H_{5}$ \\
\hline$M=M_{0}$ & II & I \\
$M=F e$ & II &
\end{tabular}

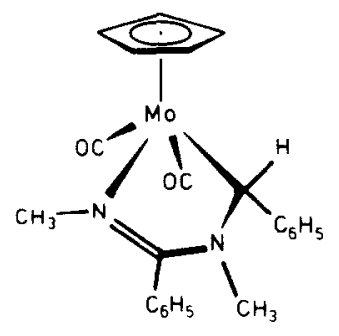

$\mathrm{B}$

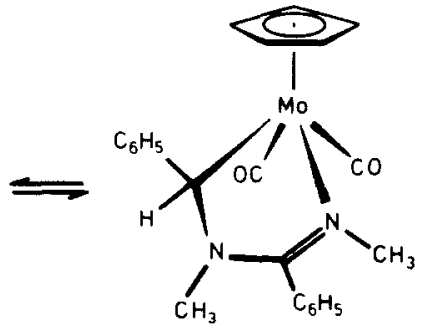

$A$ 


\section{Experimentelles}

Arbeitsvorschrift. Die Suspension von $0.50 \mathrm{~g}(0.84 \mathrm{mmol}) \mathrm{I}$ in $100 \mathrm{ml}$ THF wird mit 1.67 mmol einer $4 M$ Phenylmagnesiumbromidlösung versetzt und $17 \mathrm{~h} \mathrm{am}$ Rückfluss erhitzt. Nach dem Entfernen des Lösungsmittels löst man den öligen Rückstand in $10 \mathrm{ml}$ Benzol und chromatographiert an $\mathrm{SiO}_{2}$. Das Produkt wird als rote Zone in 20\% Ausbeute isoliert. Umkristallisation aus Ether/Pentan ergibt dunkelrote Prismen. Analyse: Gef.: C, 65.50; H, 4.93; Molgew. (massenspektroskopisch) 532 (bez. ${ }^{98} \mathrm{Mo}$ ). $\mathrm{C}_{29} \mathrm{H}_{26} \mathrm{MoN}_{2} \mathrm{O}_{2}$, ber.: C, 65.65; H, 4.94\%; Molgew. 530.2 .

Röntgenstrukturanalyse. Die Sammlung und Verarbeitung der Daten erfolgte nach bereits beschriebenem Muster [5] an einem NONIUS CAD-4-Diffraktometer mit OS/4 Software. Die kristallographischen Daten sind in Tab. 1 zusammengefasst. Atomparameter, Bindungslängen, Winkel und beste Ebenen finden sich in den Tabellen 2-5. Die Lösung der Struktur erfolgte nach der Pattersonmethode, die Lokalisierung der Nichtwasserstoffatome erfolgte direkt. Alle H-Atome wurden auf idealisierte Positionen festgesetzt $(\mathrm{C}-\mathrm{H} 0.95 \AA)$. Die $\mathrm{Cp}$ - und Phenylringe wurden als starre Körper behandelt (Cp: C-C $1.420 \AA \mathrm{C}_{6} \mathrm{H}_{5}: \mathrm{C}-\mathrm{C} 1.395 \AA$ ). Die Verfeinerung konvergierte gegen ein $R_{k}$ von $4.8 \%$ ( $R$ 5.1\%).

\section{'H-NMR-Spektren}

Die 'H-NMR-Spektren von III wurden an einem Multikern-WH 400-BrukerSpektrometer vermessen. Das $400 \mathrm{MHz}$-Gerät ist mit einem Aspekt 2000 Computer mit $80 \mathrm{~K}$ Memory, einem schnellen Pulsprogrammer und einer $96 \mathrm{M}$ Byte CDCDoppelplatte bestückt. Alle Aufnahmen wurden mit Quadratur-Detection und VierPhasen-Cyclen durchgeführt. Die Spektren wurden mit $32 \mathrm{~K}$ Datenpunkten und einer Spektralweite von $4000 \mathrm{~Hz}$ aufgenommen; dies entspricht ciner Aufnahmezeit

\section{TABELLE 1}

\section{KRISTALLDATEN}

$\begin{array}{ll}\text { Raumgruppe } & P 2_{1} / c \\ \text { Gitterkonstanten } & a 11.803(5) \AA \\ & b 12.114(6) \AA \\ & c 17.198(6) \AA \\ & \beta 96.61(3)^{\circ} \\ \text { Zellvolumen } & V 2431.2 \AA^{3} \\ \text { Surnmenformel } & \mathrm{MoN}_{2} \mathrm{O}_{2} \mathrm{C}_{29} \mathrm{H}_{26} \\ \text { Molgewicht } & 530.17 \\ \text { Moleküle/Zelle } & Z=4 \\ \text { Dichte } & 1.45 \mathrm{~g} \mathrm{~cm}^{-3} \\ \text { Absorptionskoeffizient } & 4.95 \mathrm{~cm}^{-1} \\ \text { Strahlung }\left(\text { Mo- } K_{\alpha}\right) & \lambda 0.71073 \AA \\ \text { Messbereich } & 4^{\circ} \leq 2 \theta \leq 50^{\circ} \\ \text { Scanbreite } & \Delta \theta=(0.95+0.30 \tan \theta)^{\circ} \\ \text { Registriergeschwindigkeit } & 0.48 \mathrm{bis} 6.70^{\circ} \min ^{-1} \\ \text { Gemessene Reflexe } & 4439 \\ \text { Unabhängige Reflexe }(I>3 \sigma(I)) & 2979 \\ R=\Sigma\left(F_{0}-F_{\mathrm{c}}\right) / \Sigma F_{0} & 5.1 \\ R=\left[\Sigma w\left(F_{0}-F_{\mathrm{c}}\right)^{2} / \Sigma w F_{0}^{2}\right]^{1 / 2} & 4.8 \\ \text { Wichtung } & w=\sigma(F)^{-2}\end{array}$


von $4.096 \mathrm{~s}$ und einer digitalen Auflösung von $0.244 \mathrm{~Hz}$. Die Kern-Overhauser-Differenzspektren wurden von verdünnten Lösungen in III in perdeuteriertem Chloroform aufgenommen (ca. $1 \% \mathrm{v} / \mathrm{v}, 5 \mathrm{~mm}$ Präzessionsröhrchen unter Argon abgeschmolzen). Acht Interferogramme wurden unmittelbar nach einer Bestrahlung von 10-15 s Dauer bei einer Decoupler-Off-Resonanzfrequenz aufaddiert. Anschliessend wurden acht Durchläufe bei einer Decoupler-On-Resonanzfrequenz von den ersten acht substrahiert. Dieser 16-Scan-Zyklus wurde mehrmals (20- bis 60mal) wiederholt. Dies führte zu 320 bis 960 Akkumulationen pro gesättigtem Proton. Der so erhaltene FID wurde mit Gewichtsfunktionen und gespeicherten Phasenkorrekturwerten wie ein normales Fourier-Spektrum transformiert. Kontrollmessungen an nicht-Overhauser-erhöhten Signalen zeigen, dass infolge von Feld- und Frequenzstabilität eine Abziehgenauigkeit von weit besser als $99.5 \%$ erreicht werden kann. Dies bedeutet, dass Overhauser-Erhöhungen von weniger als $0.5 \%$ sicher erkannt werden können. Die gewünschte Selektivität der Bestrahlung erfordert bei benachbarten Signalen (z. B. 7 und $7^{\prime}$ ) Leistungen für das Decoupler-Feld, die unterhalb der Sättigung liegen. Die hier beschriebenen NOEDS-Messungen wurden bei Leistungen zwischen $20 \mathrm{~dB}$ und $50 \mathrm{~dB}$ unter 0.1 Watt ausgeführt. Da das Spektrometer unter vollständiger Computer-Kontrollc ist, konnten NOEDSSequenzen bei Bestrahlung verschiedener Protonen mit verschiedenen Leistungen automatisch nacheinander ausgeführt werden. Infolge der dabei erreichten unterschiedlichen Sättigungsgrade einzelner Protonen sind die relativen Verstärkungsfaktoren nicht vergleichbar und daher im Text nicht mit aufgeführt.

Charakteristische chemische Verschiebungen: IIIA $\delta(1) 2.80, \delta(7) 2.58, \delta(8) 5.65$, $\delta(12) 4.90 \mathrm{ppm}$; IIIB $\delta\left(1^{\prime}\right) 2.88, \delta\left(7^{\prime}\right) 2.54, \delta\left(8^{\prime}\right) 5.50, \delta\left(12^{\prime}\right) 5.39 \mathrm{ppm}$; Lösungsmittel $\mathrm{CDCl}_{3}(\approx 1 \% \mathrm{v} / \mathrm{v})$, Referenz TMS intern.

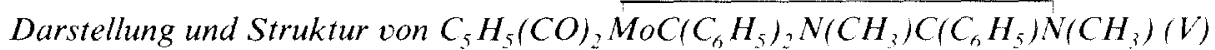

Die Umsetzung des $\mathrm{PF}_{6}^{-}$-Salzes I mit $\mathrm{C}_{6} \mathrm{H}_{5} \mathrm{MgBr}$ erfolgt in THF bei $60^{\circ} \mathrm{C}$ (Schema 1). Der sich dabei bildende rotbraune Komplex $V$ enthält in seinem IR-Spektrum zwei $\nu(\mathrm{CO})$-Frequenzen bei 1932 und $1827 \mathrm{~cm}^{-1}\left(\mathrm{CH}_{2} \mathrm{Cl}_{2}\right)$ und eine $\nu(\mathrm{CN})$-Frequenz bei $1587 \mathrm{~cm}^{-1}$. Im ${ }^{1} \mathrm{H}-\mathrm{NMR}$-Spektrum ( $\mathrm{CDCl}_{3}$ - $\mathrm{Lösung}$ ) finden sich Singuletts bei 2.13 und $2.87 \mathrm{ppm}\left(\mathrm{CH}_{3}\right)$ und $4.81 \mathrm{ppm}\left(\mathrm{C}_{5} \mathrm{H}_{5}\right)$ sowie ein Multiplett bei $7.36 \mathrm{ppm}$. Die Verbindung lehnt sich damit in ihren spektroskopischen Eigenschaften eng an die eingangs erwähnten Hydridaddukte an [2].

Die Geometrie des Moleküls (Fig. 1) ist von der quadratischen Pyramide abgeleitet [6]. Das Mo-Atom liegt $0.995 \AA$ über der leicht verzerten Grundfläche, die aus $C(6), C(7), N(1)$ und $C(8)$ gebildet wird. Der ursprünglich planare Chelatfünfring [7] ist leicht gewellt, wobei die an $\mathrm{N}(1)$ befindliche Methylgruppe vom Cp-Rest weggerichtet ist (Fig. 2).

Auffallend ist die dichte Packung der $\mathrm{Cp}$ - und der Phenylringe, was die relativ schwache Ausbeute an $\mathrm{V}$ erklären mag und andererseits die mit Hilfe des KernOverhauser-Effekts gemachten Beobachtung erhärtet. Aufgrund von Modellbetrachtungen lässt sich leicht zeigen, dass der Phenylring $C(12)-C(17)$ in einer Position weitgehend fixiert ist, in der seine Breitseite gegen den Rand des Cp-Ringes weist. Bei dem Versuch einer Rotation um die Bindung C(8)-C(12) kämen dic orthoständigen Protonen $\mathrm{H}(13)$ und $\mathrm{H}(17)$ in Kontakt mit den Cp-Wasserstoffen, die bereits in der günstigsten Anordnung nur ca. $2.2 \mathrm{~A}$ von $\mathrm{H}(13)$ und $\mathrm{H}(17)$ entfernt sind. 


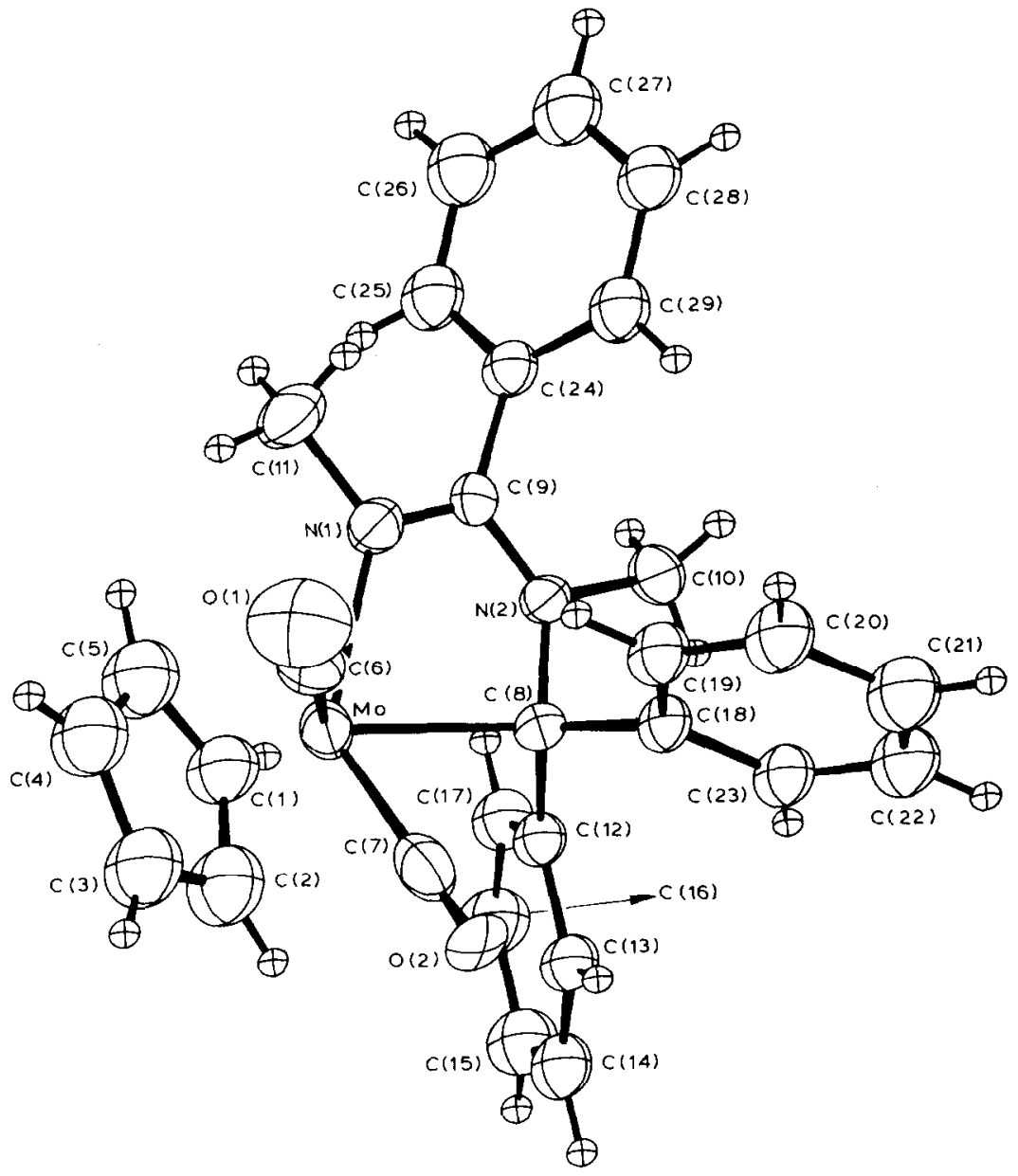

Fig. 1. Molekülstruktur von III.
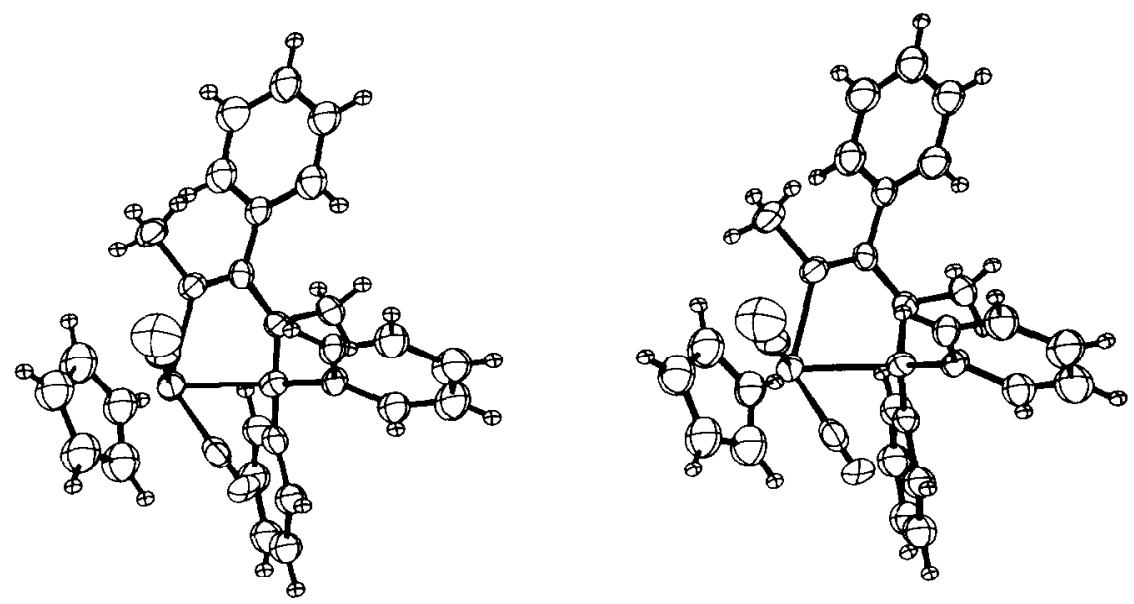

Fig. 2. Stereoansicht von Molekül III. 
TABELLE 2

ATOMKOORDINATEN UND THERMISCHE PARAMETER $(\times 1000 ;$ Mo $\times 10,000)$

\begin{tabular}{|c|c|c|c|c|c|c|c|c|c|}
\hline Atom & $x / a$ & $y / b$ & $z / c$ & $U_{11}$ & $U_{22}$ & $U_{33}$ & $U_{12}$ & $U_{13}$ & $U_{23}$ \\
\hline Mo & $0.90303(4)$ & $0.83332(4)$ & $0.37937(3)$ & $264(2)$ & $326(3)$ & $431(3)$ & $-25(3)$ & $69(2)$ & $-45(3)$ \\
\hline$N(1)$ & $0.8523(4)$ & $0.6631(4)$ & $0.3889(3)$ & $33(3)$ & $31(3)$ & $49(3)$ & $4(3)$ & $-2(2)$ & $-1(3)$ \\
\hline$N(2)$ & $0.6946(4)$ & $0.6991(4)$ & $0.2993(3)$ & $29(3)$ & $27(3)$ & $38(3)$ & $-1(2)$ & $-1(2)$ & $-1(2)$ \\
\hline$O(1)$ & $0.9432(4)$ & $0.8236(5)$ & $0.5620(3)$ & $100(4)$ & $72(4)$ & $42(3)$ & $-7(3)$ & $-4(3)$ & $2(3)$ \\
\hline$O(2)$ & $0.7989(4)$ & $1.0478(4)$ & $0.4400(3)$ & $41(3)$ & $18(3)$ & $49(4)$ & $8(2)$ & $7(3)$ & $-19(3)$ \\
\hline$C(6)$ & $0.927 l(5)$ & $0.8259(6)$ & $0.4934(4)$ & $44(4)$ & $37(4)$ & $64(5)$ & $-6(4)$ & $8(4)$ & $-3(4)$ \\
\hline$C(7)$ & $0.8345(5)$ & $0.9723(6)$ & $0.4156(4)$ & $32(4)$ & $55(5)$ & $51(5)$ & $-17(3)$ & $3(3)$ & $14(4)$ \\
\hline$C(8)$ & $0.7116(4)$ & $0.8196(5)$ & $0.3178(3)$ & $29(3)$ & $25(5)$ & $36(4)$ & $1(3)$ & $6(3)$ & $0(3)$ \\
\hline$C(9)$ & $0.7596(5)$ & $0.6271(5)$ & $0.3469(3)$ & $28(3)$ & $32(4)$ & $39(4)$ & $1(3)$ & $12(3)$ & $0(3)$ \\
\hline$C(10)$ & $0.5864(5)$ & $0.6637(5)$ & $0.2521(3)$ & $36(3)$ & $40(4)$ & $51(4)$ & $-6(3)$ & $-6(3)$ & $-2(4)$ \\
\hline$C(11)$ & $0.9247(5)$ & $0.5858(5)$ & $0.4420(4)$ & $50(4)$ & $43(4)$ & $77(6)$ & $7(4)$ & $-19(4)$ & $11(4)$ \\
\hline$C(1)$ & $0.9812(4)$ & $0.8103(4)$ & $0.2603(2)$ & $55(2)$ & & & & & \\
\hline$C(2)$ & $0.9867(4)$ & $0.9227(4)$ & $0.2840(2)$ & $64(2)$ & & & & & \\
\hline$C(3)$ & $1.0622(4)$ & $0.9303(4)$ & $0.3563(2)$ & $66(2)$ & & & & & \\
\hline$C(4)$ & $1.1034(4)$ & $0.8225(4)$ & $0.3773(2)$ & $67(2)$ & & & & & \\
\hline$C(5)$ & $1.0533(4)$ & $0.7484(4)$ & $0.3179(2)$ & $65(2)$ & & & & & \\
\hline$C(12)$ & $0.7052(3)$ & $0.8380(2)$ & $0.2423(2)$ & $31(1)$ & & & & & \\
\hline$C(13)$ & $0.6881(3)$ & $1.0017(2)$ & $0.2463(2)$ & $39(2)$ & & & & & \\
\hline$C(14)$ & $0.6908(3)$ & $1.0672(2)$ & $0.1799(2)$ & $45(2)$ & & & & & \\
\hline$C(15)$ & $0.7104(3)$ & $1.0190(2)$ & $0.1094(2)$ & $54(2)$ & & & & & \\
\hline$C(16)$ & $0.7274(3)$ & $0.9053(2)$ & $0.1054(2)$ & $53(2)$ & & & & & \\
\hline$C(17)$ & $0.7248(3)$ & $0.8398(2)$ & $0.1719(2)$ & $42(2)$ & & & & & \\
\hline$C(18)$ & $0.6150(3)$ & $0.8466(3)$ & $0.3667(2)$ & $30(1)$ & & & & & \\
\hline$C(19)$ & $0.6315(3)$ & $0.8216(3)$ & $0.4468(2)$ & $37(1)$ & & & & & \\
\hline$C(20)$ & $0.5420(3)$ & $0.8356(3)$ & $0.4905(2)$ & $48(2)$ & & & & & \\
\hline$C(21)$ & $0.4360(3)$ & $0.8746(3)$ & $0.4541(2)$ & $52(2)$ & & & & & \\
\hline$C(22)$ & $0.4195(3)$ & $0.8995(3)$ & $0.3740(2)$ & $51(2)$ & & & & & \\
\hline$C(23)$ & $0.5090(3)$ & $0.8855(3)$ & $0.3303(2)$ & $39(2)$ & & & & & \\
\hline
\end{tabular}




\begin{tabular}{|c|c|c|c|c|}
\hline$C(24)$ & $0.7194(3)$ & $0.5121(3)$ & $0.3506(2)$ & $33(1)$ \\
\hline$C(25)$ & $0.7777(3)$ & $0.4251(3)$ & $0.3211(2)$ & $47(2)$ \\
\hline$C(26)$ & $0.7464(3)$ & $0.3163(3)$ & $0.3338(2)$ & $58(2)$ \\
\hline$C(27)$ & $0.6569(3)$ & $0.2945(3)$ & $0.3761(2)$ & $52(2)$ \\
\hline$C(28)$ & $0.5986(3)$ & $0.3815(3)$ & $0.4056(2)$ & $49(2)$ \\
\hline$C(29)$ & $0.6298(3)$ & $0.4903(3)$ & $0.3929(2)$ & $41(2)$ \\
\hline $\mathbf{H}(1)$ & $0.9371(4)$ & $0.7816(4)$ & $0.2140(2)$ & $204(14)$ \\
\hline $\mathbf{H}(2)$ & $0.9469(4)$ & $0.9824(4)$ & $0.2564(2)$ & $204(14)$ \\
\hline $\mathbf{H}(3)$ & $1.0817(4)$ & $0.9959(4)$ & $0.3855(2)$ & $204(14)$ \\
\hline$H(4)$ & $1.1553(4)$ & $0.8034(4)$ & $0.4230(2)$ & $204(14)$ \\
\hline $\mathrm{H}(5)$ & $1.0659(4)$ & $0.6710(4)$ & $0.3169(2)$ & $204(14)$ \\
\hline$H(101)$ & $0.5525(5)$ & $0.7244(5)$ & $0.2223(3)$ & $71(13)$ \\
\hline$H(102)$ & $0.5357(5)$ & $0.6379(5)$ & $0.2861(3)$ & $71(13)$ \\
\hline $\mathrm{H}(\mathrm{I03})$ & $0.6011(5)$ & $0.6060(5)$ & $0.2176(3)$ & $71(13)$ \\
\hline H(111) & $0.9891(5)$ & $0.6240(5)$ & $0.4690(4)$ & $141(23)$ \\
\hline $\mathrm{H}(112)$ & $0.9500(5)$ & $0.5272(5)$ & $0.4121(4)$ & $141(23)$ \\
\hline$H(113)$ & $0.8806(5)$ & $0.5565(5)$ & $0.4791(4)$ & $141(23)$ \\
\hline $\mathbf{H}(13)$ & $0.6748(3)$ & $1.0345(2)$ & $0.2942(2)$ & $93(10)$ \\
\hline$H(14)$ & $0.6792(3)$ & $1.1446(2)$ & $0.1826(2)$ & $93(10)$ \\
\hline$H(15)$ & $0.7122(3)$ & $1.0636(2)$ & $0.0642(2)$ & $93(10)$ \\
\hline $\mathrm{H}(16)$ & $0.7408(3)$ & $0.8724(2)$ & $0.0574(2)$ & $93(10)$ \\
\hline $\mathrm{H}(17)$ & $0.7364(3)$ & $0.7624(2)$ & $0.1691(2)$ & $93(10)$ \\
\hline$H(19)$ & $0.7037(3)$ & $0.7951(3)$ & $0.4716(2)$ & $82(10)$ \\
\hline $\mathrm{H}(20)$ & $0.5533(3)$ & $0.8186(3)$ & $0.5450(2)$ & $82(10)$ \\
\hline $\mathrm{H}(21)$ & $0.3751(3)$ & $0.8841(3)$ & $0.4838(2)$ & $82(10)$ \\
\hline $\mathbf{H}(22)$ & $0.3473(3)$ & $0.9261(3)$ & $0.3491(2)$ & $82(10)$ \\
\hline $\mathbf{H}(23)$ & $0.4977(3)$ & $0.9025(3)$ & $0.2758(2)$ & $82(10)$ \\
\hline$H(25)$ & $0.8387(3)$ & $0.4400(3)$ & $0.2923(2)$ & $143(14)$ \\
\hline$H(26)$ & $0.7861(3)$ & $0.2571(3)$ & $0.3137(2)$ & $143(14)$ \\
\hline $\mathrm{H}(27)$ & $0.6356(3)$ & $0.2204(3)$ & $0.3848(2)$ & $143(14)$ \\
\hline $\mathrm{H}(28)$ & $0.5376(3)$ & $0.3666(3)$ & $0.4344(2)$ & $143(14)$ \\
\hline $\mathbf{H}(29)$ & $0.5901(3)$ & $0.5495(3)$ & $0.4129(2)$ & $143(14)$ \\
\hline
\end{tabular}


TABELLE 3

INTRAMOLEKULARE BINDUNGSLÄNGEN VON V (Ả)

\begin{tabular}{llll}
\hline Mo-C(1) & $2.386(7)$ & $\mathrm{N}(1)-\mathrm{C}(9)$ & $1.294(10)$ \\
$\mathrm{Mo}-\mathrm{C}(2)$ & $2.307(7)$ & $\mathrm{C}(9)-\mathrm{N}(2)$ & $1.352(10)$ \\
$\mathrm{Mo}-\mathrm{C}(3)$ & $2.300(7)$ & $\mathrm{N}(2)-\mathrm{C}(10)$ & $1.471(9)$ \\
$\mathrm{Mo}-\mathrm{C}(4)$ & $2.374(7)$ & $\mathrm{N}(2)-\mathrm{C}(8)$ & $1.500(10)$ \\
$\mathrm{Mo}-\mathrm{C}(5)$ & $2.426(7)$ & $\mathrm{C}(8)-\mathrm{C}(12)$ & $1.533(10)$ \\
$\mathrm{Mo}-\mathrm{C}(6)$ & $1.941(11)$ & $\mathrm{C}(8)-\mathrm{C}(18)$ & $1.550(8)$ \\
$\mathrm{Mo}-\mathrm{C}(7)$ & $2.007(11)$ & $\mathrm{C}(9)-\mathrm{C}(24)$ & $1.476(9)$ \\
$\mathrm{Mo}-\mathrm{C}(8)$ & $2.353(8)$ & Mo-Cent & $2.026(7)$ \\
Mo-N(1) & $2.160(7)$ & $\mathrm{Cp}$ (ideal) & 1.420 \\
$\mathrm{C}(6)-\mathrm{O}(1)$ & $1.168(10)$ & Phen (ideal) & 1.395 \\
$\mathrm{C}(7)-\mathrm{O}(2)$ & $1.115(11)$ & $\mathrm{C}-\mathrm{H}$ (ideal) & 0.95 \\
$\mathrm{~N}(1)-\mathrm{C}(11)$ & $1.486(10)$ & & \\
\hline
\end{tabular}

"Cent $=$ Geometrische Mitte des $\mathrm{C}_{5} \mathrm{H}_{5}$-Rings.

Aus diesem Grund sollte der $\alpha$-Phenylrest das Verhalten der Cp-Protonen im ${ }^{1} H$-NMR über die gleichen Ringstromeffekte beeinf'ussen können, wie bereits in einer Reihe von Verbindungen mit $\beta$-ständigem Phenylrest beschrieben [8]. Allerdings beträgt der Winkel zwischen den Ebenen des $\mathrm{Cp}$-Ringes und des $(\mathrm{C}(12)-\mathrm{C}(17))$ Phenylrestes $134.6^{\circ}$ (Tab. 5). Bei $\beta$-ständigen Phenylresten geht dieser Winkel eher gegen $90^{\circ}$, dafür bleibt dem Aromaten mehr Bewegungsfreiheit. Der Abstand der Zentren von $\mathrm{Cp}$ - und $\mathrm{C}_{6} \mathrm{H}_{5}$-Ring ist mit ca. $4.6 \AA$ in beiden Fällen annähernd gleich. Der weniger günstige Winkel in $V$ kann somit durch eine längere Verweilzeit der $\mathrm{Cp}$-Protonen im Anisotropiekegel des benachbarten $\alpha$-Phenylrestes kompensiert werden.

\section{${ }^{I} \mathrm{H}$-NMR-spektroskopische Untersuchungen an $\mathrm{C}_{5} \mathrm{H}_{5}(\mathrm{CO}), \overline{\mathrm{MOCH}\left(\mathrm{C}_{6} \mathrm{H}_{5}\right)}$ - $\overrightarrow{\mathrm{N}\left(\mathrm{CH}_{3}\right) \mathrm{C}\left(\mathrm{C}_{6} \mathrm{H}_{5}\right) \mathrm{NCH}}(\mathrm{III})$}

Fig. 3 zeigt das $400 \mathrm{MHz}{ }^{~} \mathrm{H}-\mathrm{NMR}$-Spektrum eines Isomerengemisches IIIA/IIIB

\section{TABELLE 4}

INTRAMOI.FKUIAARE BINDUNGSWINKEL VON V $\left(^{\circ}\right)$

\begin{tabular}{|c|c|c|c|}
\hline $\mathrm{C}(6)-\mathrm{Mo}-\mathrm{C}(7)$ & $74.3(2)$ & $C(12)-C(8)-C(18)$ & $113.5(5)$ \\
\hline $\mathrm{C}(6)-\mathrm{M}_{0}-\mathrm{C}(8)$ & $115.9(2)$ & $\mathrm{C}(8)-\mathrm{N}(2)-\mathrm{C}(10)$ & $118.6(7)$ \\
\hline $\mathrm{C}(6)-\mathrm{Mo}-\mathrm{N}(1)$ & $83.1(2)$ & $C(9)-N(2)-C(10)$ & $120.8(7)$ \\
\hline$C(7)-M_{\circ}-C(8)$ & $78.2(2)$ & $\mathrm{N}(2)-\mathrm{C}(9)-\mathrm{C}(24)$ & $118.6(7)$ \\
\hline$C(7)-M_{0}-N(1)$ & $130.4(2)$ & $\mathrm{N}(1)-\mathrm{C}(9)-\mathrm{C}(24)$ & $123.0(8)$ \\
\hline$C(8)-M o-N(1)$ & $73.0(2)$ & $C(9)-N(1)-C(11)$ & $119.4(8)$ \\
\hline $\mathrm{Mo}-\mathrm{C}(8)-\mathrm{N}(2)$ & $104.7(5)$ & $\mathrm{Mo}-\mathrm{N}(1)-\mathrm{C}(11)$ & $120.5(5)$ \\
\hline $\mathrm{C}(8)-\mathrm{N}(2)-\mathrm{C}(9)$ & $117.0(7)$ & $\mathrm{Mo}-\mathrm{C}(6)-\mathrm{O}(1)$ & $178.4(9)$ \\
\hline$N(2)-C(9)-N(1)$ & $118.4(8)$ & $\mathrm{Mo}-\mathrm{C}(7)-\mathrm{O}(2)$ & $176.1(9)$ \\
\hline $\mathrm{C}(9)-\mathrm{N}(1)-\mathrm{Mo}$ & $120.0(6)$ & $\mathrm{C}(6)-\mathrm{Mo}-\mathrm{Cent}{ }^{a}$ & $120.8(2)$ \\
\hline $\mathrm{Mo}-\mathrm{C}(8)-\mathrm{C}(12)$ & $105.4(4)$ & $\mathrm{C}(7)-\mathrm{Mo}-\mathrm{Cent}$ & $118.3(2)$ \\
\hline $\mathrm{Mo}-\mathrm{C}(8)-\mathrm{C}(18)$ & $118.4(4)$ & $\mathrm{N}(1)-\mathrm{M}_{0}-\mathrm{Cent}$ & $111.3(2)$ \\
\hline $\mathrm{N}(2)-\mathrm{C}(8)-\mathrm{C}(12)$ & $111.0(5)$ & $\mathrm{C}(8)-\mathrm{Mo}_{0}-\mathrm{Cent}$ & $123,2(2)$ \\
\hline$N(2)-C(8)-C(18)$ & $103.5(5)$ & & \\
\hline
\end{tabular}

${ }^{a}$ Cent $=$ Mitte des $\mathrm{C}_{5} \mathrm{H}_{5}$-Rings. 
TABELLE 5

BESTE EBENEN UND DIEDERWINKEL VON V
A. $\mathrm{C}(1)-\mathrm{C}(5)$
$0.853 x+0.146 y-0.500 z-8.532=0$
Mo -2.022
B. $\mathrm{C}(12)-\mathrm{C}(17)$
$-0.955 x-0.133 y-0.265 z+9.876=0$
C. $\mathrm{C}(18)-\mathrm{C}(23)$
$-0.270 x-0.939 y-0.211 z-12.655=0$
D. $\mathrm{C}(24)-\mathrm{C}(29)$
$-0.528 x+0.021 y-0.849 z+8.935=0$

E. $\quad \mathrm{Mo}, \mathrm{N}(1), \mathrm{N}(2), \mathrm{C}(8), \mathrm{C}(9)$

$0.557 x-C .126 y-0.821 z+1.289=0$

$\begin{array}{lrlr}\text { Mo } & 0.114 & \mathrm{C}(10) & 0.250 \\ \mathrm{~N}(1) & -0.106 & \mathrm{C}(11) & -0.330 \\ \mathrm{~N}(2) & 0.181 & \mathrm{C}(12) & 0.841 \\ \mathrm{C}(8) & -0.175 & \mathrm{C}(18) & -1.605 \\ \mathrm{C}(9) & -0.014 & \mathrm{C}(24) & -0.160\end{array}$

F. $\mathrm{C}(6), \mathrm{C}(7), \mathrm{N}(1), \mathrm{C}(8)$
$0.807 x+0.134 y-0.575 z-4.461=0$

$\begin{array}{lrlr}C(6) & -0.140 & \mathrm{~N}(1) & 0.123 \\ \mathrm{C}(7) & 0.137 & \mathrm{C}(8) & -0.121 \\ \text { Mo } & 0.995 & \mathrm{Cent} & 3.010\end{array}$

Diederwinkel $\left({ }^{\circ}\right)$

$\begin{array}{llrllr}\text { A } & \text { B } & 134.6 & \text { E } & \text { B } & 107.3 \\ \text { A } & \text { C } & 105.2 & \text { E } & \text { C } & 81.9 \\ \text { A } & \text { D } & 91.3 & \text { E } & \text { D } & 66.4 \\ \text { A } & \text { E } & 29.8 & \text { E } & \text { F } & 25.2 \\ \text { A } & \text { F } & 5.1 & & & \end{array}$
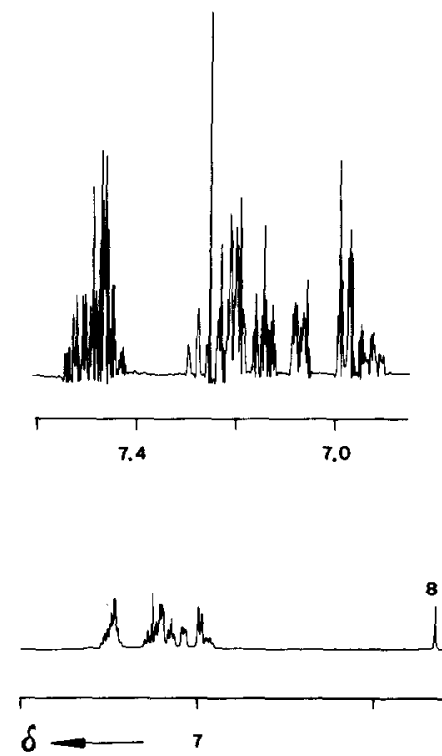

Fig. 3. $400 \mathrm{MHz}{ }^{1} \mathrm{H}-\mathrm{NMR}-S p e k t r u m$ von III in $\mathrm{CDCl}_{3}$. Oben Aromatenbereich gedehnt und Linienverschärfung infolge Lorentz-Gauss Transformation.
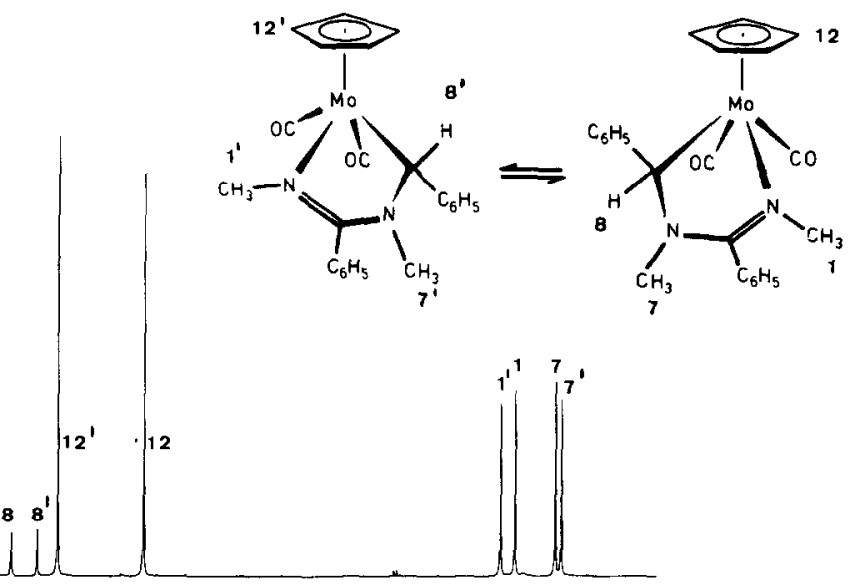


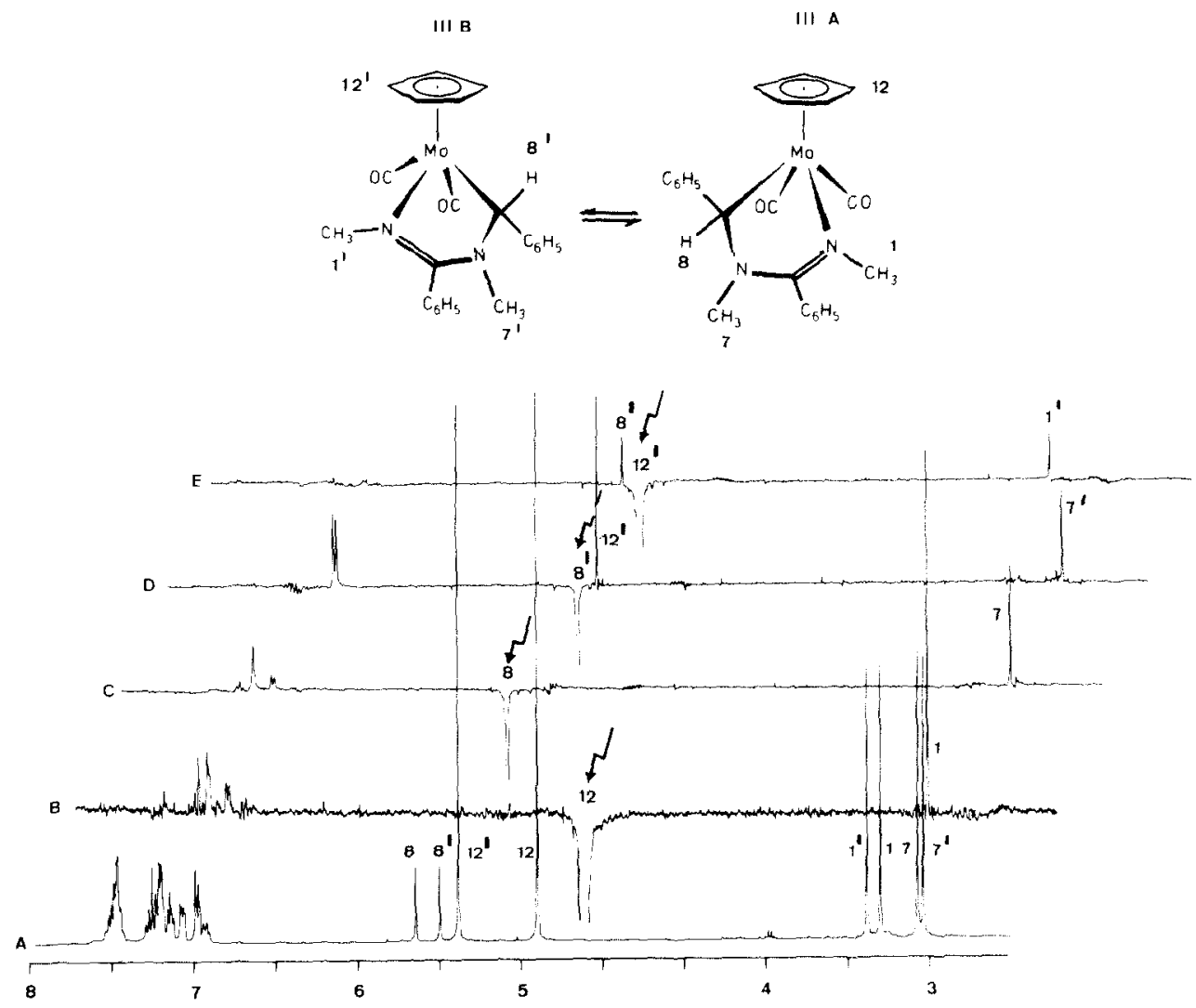

Fig. 4. $400 \mathrm{MHz}$ 'II-NMR-Spektren von III. A normales Spektrum, B-E Kern-Overhauser-DiffcrenzSpektren bei Sättigung von $12 \mathrm{~B}, 8 \mathrm{C}, 8^{\prime} \mathrm{D}$ und $12^{\prime} \mathrm{E}$. In den Differenzspektren erscheinen Overhausererhöhte Signale selektiv als positive Signale.

in $\mathrm{CDCl}_{3}$. Die Zuordnung der Signale (Tab. 1) lässt sich anhand der säulenchromatographisch getrennten reinen Isomeren treffen [1], wobei als Isomer A (vgl. Fig. 3) die Verbindung mit dem $\mathrm{C}_{5} \mathrm{H}_{5}$-Signal bei höherem Feld bezeichnet wird. Die Aufspaltung der Signale für die Phenylprotonen ist selbst bei $400 \mathrm{MHz}$ kompliziert und wird im einzelnen nicht analysiert.

In Fig. 4 sind einige NOE-Differenz-Spektren (B-E) zusammen mit dem normalen Spektrum (A) von III dargestellt. Die Skala bezieht sich dabei auf Spektrum A; die NOE-Spektren sind als "stacked plot" nach rechts versetzt. Die DifferenzSpektren erhält man durch Subtraktion eines freien Induktionsabfalls, aufgenommen nach Sättigung eines Signals, von einem freien Induktionsabfall ohne Sättigung [4]. Dadurch erscheinen die gesättigten Protonen als Negativ- oder Emissionssignale. Positive Signale (Absorption) erhält man selektiv für räumlich benachbarte Protonen, die zu den gesättigten Protonen Dipol-Dipol-Wechselwirkung durch den Raum zeigen.

Eine Sättigung der $\mathrm{C}_{5} \mathrm{H}_{5}$-Protonen 12 (Spektrum B) ergibt positive Signale im Phenylbereich und für die $\mathrm{CH}_{3}$-Gruppe 1. Es wird kein Effekt für Proton 8 beobachtet! Sättigt man in Spektrum C Proton 8, so lassen sich Wechselwirkungen 
mit dem $\mathrm{CH}_{3}$-Rest 7 und mit Phenylprotonen nachweisen. In Spektrum D ist das Proton $8^{\prime}$ gesättigt, was Auswirkungen auf die $\mathrm{CH}_{3}$-Gruppe $7^{\prime}$ und auf den Phenylbereich hat. In Spektrum E wird bei einer Sättigung von 12' nicht nur das Proton $8^{\prime}$, sondern auch die Methylgruppe 1' verstärkt, dagegen treten keine Wechselwirkungen mit Phenylprotonen auf.

Anhand dieser Befunde wird für Isomer IIIA folgende Struktur wahrscheinlich: Das neugeschaffene Chiralitätszentrum zeichnet sich durch eine räumliche Nachbarschaft des Phenylrings zum $\mathrm{C}_{5} \mathrm{H}_{5}$-Rest aus, während der addierte Hydridwasserstoff auf der dem $\mathrm{C}_{5} \mathrm{H}_{5}$-Rest abgewandten Seite liegt, was Wechselwirkungen nur mit den benachbarten $o$-Phenyl- und $N$-Methylprotonen zulässt. Diese Anordnung erklärt auch die Hochfeldverschiebung des $\mathrm{C}_{5} \mathrm{H}_{5}$-Signals von IIIA, die dadurch zustande kommt, dass die $\mathrm{C}_{5} \mathrm{H}_{5}$-Protonen in den inneren Anisotropiebereich des Phenylrings fallen (" $\alpha$-Phenyl-Effekt").

Entsprechend liegt im Isomeren IIIB das Methinproton in Nachbarschaft zum $\mathrm{C}_{5} \mathrm{H}_{5}$-Rest. Die gleiche strukturelle Anordnung dürfte auch in den Fe-Komplexen IVA/IVB anzutreffen sein, wenn man die chemischen Verschiebungen der $\mathrm{C}_{5} \mathrm{H}_{5}$ Reste als Bezug wählt. Für den stereochemischen Ablauf der Hydridaddition an die Carbcnchelatkomplexe I und II lässt sich nunmehr aufgrund der vorliegenden Ergebnisse ein nahezu stereoselektiver Angriff von der dem $\mathrm{C}_{5} \mathrm{H}_{5}$-Rest zugewandten Seite des Chelatrings annehmen. Im Fall des am Metallatom konfigurationsstabilen Fe-Komplexes IV bildet sich dabei IVB zu 95\%. Der ursprünglich gebildete MoKomplex IIIB wandelt sich dagegen zu $85 \%$ in das thermodynamisch stabilere IIIA um. Für diese Umlagerung erscheint nach den vorliegenden Untersuchungen eine schwache Anziehung durch eine $\mathrm{C}_{5} \mathrm{H}_{5} / \mathrm{C}_{6} \mathrm{H}_{5}$-Wechselwirkung als Triebkraft. Inwieweit die Konformation des Chelatfünfrings daran beteiligt ist, kann mangels Vergleichsmaterials noch nicht entschieden werden.

\section{Literatur}

1 H. Brunner und J. Wachter, J. Organometal. Chem., 201 (1980) 453.

2 H. Brunner, J. Wachter, I. Bernal und M. Creswick, Angew. Chem., 91 (1979) 920; Angew. Chem. Int. Ed. Engl., 18 (1979) 861.

3 H. Brunner, G. Agrifoglio, R. Benn und A. Rufińska, J. Organometal. Chem., 217 (1981) 365.

4 W.E. Hull, Aspect 2000 Application Note No. 1, Bruker Report 1 (1978) 4; R. Benn, A. Rufińska und G. Schroth, J. Organometal. Chem., 217 (1981) 91.

5 G.M. Reisner, I. Bernal und G.R. Dobson, J. Organometal. Chem., 157 (1978) 23.

6 P. Kubácek, R. Hoffmann und Z. Havlas, Organometallics, 1 (1982) 180 und dort zit. Lit.

7 M.F. Lappert und A.J. Oliver, J. Chem. Soc. Dalton, (1974) 65.

8 H. Brunner, G. Agrifoglio, I. Bernal und M.W. Creswick, Angew. Chem., 92 (1980) 645; Angew. Chem. Int. Ed. Engl., 19 (1980) 641. 